\title{
A Modified Equation Approach to Selecting a Nonstandard Finite Difference Scheme Applied to the Regularized Long Wave Equation
}

\author{
E. Momoniat \\ Centre for Differential Equations, Continuum Mechanics and Applications, School of Computational and Applied Mathematics, \\ University of the Witwatersrand, Private Bag 3, Johannesburg 2050, South Africa
}

Correspondence should be addressed to E. Momoniat; ebrahim.momoniat@wits.ac.za

Received 11 February 2014; Accepted 7 April 2014; Published 29 April 2014

Academic Editor: Rehana Naz

Copyright (C) 2014 E. Momoniat. This is an open access article distributed under the Creative Commons Attribution License, which permits unrestricted use, distribution, and reproduction in any medium, provided the original work is properly cited.

\begin{abstract}
Two nonstandard finite difference schemes are derived to solve the regularized long wave equation. The criteria for choosing the "best" nonstandard approximation to the nonlinear term in the regularized long wave equation come from considering the modified equation. The two "best" nonstandard numerical schemes are shown to preserve conserved quantities when compared to an implicit scheme in which the nonlinear term is approximated in the usual way. Comparisons to the single solitary wave solution show significantly better results, measured in the $L_{2}$ and $L_{\infty}$ norms, when compared to results obtained using a Petrov-Galerkin finite element method and a splitted quadratic B-spline collocation method. The growth in the error when simulating the single solitary wave solution using the two "best" nonstandard numerical schemes is shown to be linear implying the nonstandard finite difference schemes are conservative. The formation of an undular bore for both steep and shallow initial profiles is captured without the formation of numerical instabilities.
\end{abstract}

\section{Introduction}

In this paper we derive two nonstandard finite difference schemes to solve the regularized long wave (RLW) equation. The RLW equation has been derived by Peregrine [1] to model the propagation of a unidirectional weakly nonlinear and weakly dispersive water wave known as an undular bore. The model equation is given by the mixed space-time partial differential equation:

$$
\frac{\partial u}{\partial t}-\frac{\partial^{3} u}{\partial t \partial x^{2}}+\frac{\partial u}{\partial x}+u \frac{\partial u}{\partial x}=0, \quad x \in \mathbb{R}, t>0,
$$

where

$$
u(x, 0)=g(x), \quad x \in \mathbb{R} .
$$

The RLW equation (1) satisfies the Dirichlet boundary conditions

$$
u \longrightarrow 0, \quad x \pm \infty
$$

Benjamin et al. [2] have proposed the RLW equation (1) as an alternative to the Korteweg de Vries (KdV) equation [3]

$$
\frac{\partial u}{\partial t}+\frac{\partial u}{\partial x}+u \frac{\partial u}{\partial x}+\frac{\partial^{3} u}{\partial x^{3}}=0
$$

to model phenomena in wave propagation and other physical systems. The criteria for selecting the "best" nonstandard approximation to the nonlinear term in the RLW equation come from considering the effects of the modified equation on the single solitary wave solution admitted by the RLW equation (1). The modified equation is obtained by considering coefficients $\mathcal{O}\left(\Delta t / \Delta x^{2}\right)$ of a Taylor series expansion of an implicit scheme and nonstandard finite difference schemes approximating the RLW equation. We show that the two "best" nonstandard approximations to the nonlinear term make the nonstandard finite difference schemes approximating the RLW equation (1) second-order accurate, that is, $\mathcal{O}\left(\Delta t^{2} / \Delta x^{4}\right)$, where $\Delta t$ is the time step length and $\Delta x$ is the spatial step length. To the best of our knowledge this is the first paper in which the analysis of the modified equation has 
been used to justify the choice of nonstandard approximation to a nonlinear term.

The nonstandard finite difference schemes are reduced to linear tridiagonal systems that are solved using a Thomas algorithm [4]. We show that the nonstandard numerical schemes preserve the conserved quantities (these quantities have been obtained by Olver [5]):

$$
\begin{aligned}
& I_{1}=\int_{-\infty}^{\infty} u d x \\
& I_{2}=\int_{-\infty}^{\infty}\left(u^{2}+\left(u_{x}\right)^{2}\right) d x, \\
& I_{3}=\int_{-\infty}^{\infty}\left(u^{3}+3 u^{2}\right) d x .
\end{aligned}
$$

For completeness, we show that a standard implicit scheme in which the nonlinear term is approximated in the usual way does not preserve (5)-(7). Numerical solutions obtained from simulations of the nonstandard finite difference schemes modelling the single solitary wave solution [1] of the RLW equation (1) given by

$$
u(x, t)=3 v \operatorname{sech}^{2}\left(k_{0} x+k_{1} t+x_{0}\right),
$$

where

$$
k_{0}=\frac{1}{2} \sqrt{\frac{v}{1+v}}, \quad k_{1}=-\frac{1}{2} \sqrt{v(1+v)},
$$

and $x_{0}$ is an arbitrary constant, show significantly better results when measured in the $L_{2}$ and $L_{\infty}$ norms when compared to simulations of the single solitary wave solution using a Petrov-Galerkin finite element method [6] and a splitted quadratic B-spline collocation method [7]. We also show that a nonstandard finite difference scheme is able to capture the formation of an undular bore for both steep and shallow initial profiles without the formation of numerical instabilities on the solution profile.

Various numerical approaches have been developed to solve the RLW equation (1). Peregrine [1] implemented a finite difference scheme to solve (1) that is first-order accurate in time and second-order accurate in space. Eilbeck and McGuire [8, 9] have derived a three-level second-order accurate space and time scheme. An implicit finite difference scheme has been implemented by Kutluay and Esen [10]. Jain et al. [11] have developed a numerical scheme that is secondorder accurate in both space and time based on cubic spline interpolations. Bona et al. [12] review fourth-order finite difference schemes to solve (1). Numerical methods based on spline Galerkin techniques have also been implemented [7, 13-19]. An analysis of finite element techniques applied to the RLW equation has been undertaken by Luo and Liu [20]. Pseudospectral methods have been developed by Ben-yu and Manoranjan [21] and Sloan [22] with extensions to Chebyshev collocation methods investigated by Ali [23]. Avilez-Valente and Seabra-Santos [6] show that their approach using a Petrov-Galerkin finite element method outperforms numerical methods based on spline Galerkin as well as pseudospectral methods but is equivalent to the splitted quadratic B-spline collocation method introduced by Dağ et al. [7]. Avilez-Valente and Seabra-Santos [6] use finite elements in both time and space. They introduce additional streamline upwind terms in the weight functions to ensure that dispersion correction and selective dissipation occur. Two versions of the numerical scheme are derived. A nonlinear version of the resulting predictor-corrector scheme is found to be third-order accurate. A linear version of the resulting scheme is found to be fourth-order accurate. Both versions of the numerical scheme are implicit and conditionally stable. Raslan [24] has employed a collocation method based on a cubic B-spline method to solve the RLW equation. Bhardwaj and Shankar [25] developed a numerical technique based on quintic splines. Cubic [26], quartic [27], and quintic $[28,29]$ B-spline collocation methods applied to a splitted RLW equation have been investigated to solve (1). Irk [30] extends the work of Bhardwaj and Shankar [25] by considering an Adams-Moulton time-integration scheme coupled with a quintic spline collocation method for the spatial variable. Araújo and Durán [31] have discussed the importance of using numerical schemes that conserve the invariants of (1). More specifically, they show that the error growth in schemes that are conservative is linear whilst nonconservative schemes have quadratic growth in the errors. Araújo and Durán [31] base their results on the fact that the RLW equation admits a Hamiltonian structure. Also, one of its invariants is generated by a symmetry group. Solitary wave solutions admitted by the RLW equation then occur as critical points of the Hamiltonian function [31]. This geometric interpretation of the solitary wave solution is used to determine the relationship between numerical schemes that conserve quantities (5)-(7) and the linear growth in error. Cai derives the Bridge's multisymplectic form of the RLW equation to derive a ten-point multisymplectic scheme to solve the RLW equation [32].

Avilez-Valente and Seabra-Santos [6] perform a thorough comparison of their results with the work of Dağ [14], Dağ et al. [7], Gardner et al. [17], Gardner et al. [18], and Jain et al. [11]. Avilez-Valente and Seabra-Santos [6] conclude that their Petrov-Galerkin finite element formulation and the splitted quadratic B-spline collocation method implemented by Dağ et al. [7] are the most effective approaches for solving the RLW equation. We compare our results to the results obtained by Avilez-Valente and Seabra-Santos [6] and Dağ et al. [7]. We show the results obtained by Avilez-Valente and Seabra-Santos [6] and Dağ et al. [7] in Tables 1 and 2, respectively. In Tables 1 and 2 the $L_{2}$ and $L_{\infty}$ norms are given by

$$
\begin{aligned}
& L_{2}=\left|u_{i}^{j+1}-u^{*}\left(x_{i}, t_{j}\right)\right|_{2}=\left(\Delta x \sum_{i=0}^{n}\left|u_{i}^{j+1}-u^{*}\left(x_{i}, t_{j}\right)\right|^{2}\right)^{1 / 2}, \\
& L_{\infty}=\left|u_{i}^{j+1}-u^{*}\left(x_{i}, t_{j}\right)\right|_{\infty}=\max _{i}\left|u_{i}^{j+1}-u^{*}\left(x_{i}, t_{j}\right)\right|,
\end{aligned}
$$

where $u^{*}\left(x_{i}, t_{j}\right)$ is the value of the single solitary wave solution (8) to the RLW equation at $x=x_{i}$ and $t=t_{j}$ for $v=0.1$ and $x_{0}=0$. 
Table 1: Table of values obtained by Avilez-Valente and Seabra-Santos [6] using a Petrov-Galerkin finite element method.

\begin{tabular}{|c|c|c|c|c|c|}
\hline Time & $I_{1}$ & $I_{2}$ & $I_{3}$ & $L_{2} \times 10^{3}$ & $L_{\infty} \times 10^{3}$ \\
\hline 0 & 3.979927 & 0.8104625 & 2.579007 & 0.00000 & 0.00000 \\
\hline 4 & 3.979944 & 0.8104625 & 2.579007 & 0.01549 & 0.00695 \\
\hline 8 & 3.979947 & 0.8104625 & 2.579007 & 0.03000 & 0.01343 \\
\hline 12 & 3.979946 & 0.8104625 & 2.579007 & 0.04306 & 0.01868 \\
\hline 16 & 3.979939 & 0.8104625 & 2.579007 & 0.05464 & 0.02292 \\
\hline 20 & 3.979909 & 0.8104625 & 2.579007 & 0.06493 & 0.02643 \\
\hline
\end{tabular}

TABLE 2: Table of values obtained by Dağ et al. [7] using a splitted quadratic B-spline collocation method.

\begin{tabular}{lccccc}
\hline Time & $I_{1}$ & $I_{2}$ & $I_{3}$ & $L_{2} \times 10^{3}$ & $L_{\infty} \times 10^{3}$ \\
\hline 0 & 3.979926 & 0.8104646 & 2.579007 & 0.00000 & 0.00000 \\
4 & 3.979930 & 0.8104626 & 2.578998 & 0.01757 & 0.02249 \\
8 & 3.979924 & 0.8104606 & 2.578994 & 0.03355 & 0.00887 \\
12 & 3.979926 & 0.8104621 & 2.578997 & 0.04075 & 0.01072 \\
16 & 3.979918 & 0.8104624 & 2.578999 & 0.04315 & 0.01321 \\
20 & 3.979890 & 0.8104625 & 2.578999 & \\
\hline
\end{tabular}

The work undertaken in this paper is a major contribution to the field because of the accuracy of the results obtained for the low computational cost when compared to other methods. An example of the high computational cost that can be incurred to obtain accurate results can be seen in the work of Bona et al. [12]. Bona et al. [12] have implemented a Runge-Kutta approximation as a first step in a leap frog approximation to the time derivative. Bona et al. [12] approximate the spatial derivatives using a GregoryEuler-Maclaurin (GEM) scheme which has a truncation error $\mathcal{O}\left(\Delta x^{4}\right)$. Bona et al. [12] showed that by using a semidiscrete scheme they could obtain a tridiagonal system. Bona et al. [12] compare leap frog (LF), fourth-order Runge-Kutta (RK), and multistep (MS) predictor-corrector approximations to the time derivative. Fourth-order discretizations in space that lead to Störmer-Numerov (SN) type schemes [33] are also considered. Fourth-order Runge-Kutta approximations to the time derivative coupled with the SN approximation to the spatial derivative (SN-RK schemes) were simulated. The SN-RK scheme performed worse in the simulations undertaken by Bona et al. [12]. The Petrov-Galerkin finite element method of Avilez-Valente and Seabra-Santos [6] is also reduced to a tridiagonal system but has to be evaluated several times at each time step because of the high-order time integration method involved. The splitted quadratic Bspline collocation scheme implemented by Dağ et al. [7] leads to a pentadiagonal system that also has to be evaluated multiple times at each time step because of the high-order time integration method that is chosen. As mentioned earlier, the nonstandard finite difference schemes are reduced to linear tridiagonal systems that we solve using a Thomas algorithm [4]. The implicit nature of the schemes means that at each time step the system matrix is recalculated and the system resolved. This is computationally not as expensive as the methods mentioned above.
In this paper we investigate nonstandard approximations to the nonlinear term in the RLW equation to derive nonstandard finite difference schemes approximating (1). An introduction to nonstandard finite difference schemes and their applications to differential equations is given in the paper by Mickens [34]. Applications to ordinary differential equations are discussed in Mickens [35] and Anguelov and Lubuma [36]. Theoretical developments of nonstandard finite difference schemes and their applications to partial differential equations are investigated by Anguelov and Lubuma [37]. Mickens [38] considers the general application of nonstandard finite difference schemes to nonlinear reactionadvection equations. Mickens [39] extends these results by considering reaction-diffusion equations and derives positivity preserving nonstandard finite difference schemes. Jordan [40] derives a nonstandard finite difference scheme satisfying a positivity condition to model heat transfer with a quartic nonlinearity. Mickens [41] constructs nonstandard finite difference schemes to solve a Fisher partial differential equation that has application in ecology. Positivity preserving schemes for Maxwell-Cattaneo wave equations are derived by Mickens and Jordan [42].

In this paper we select the "best" nonstandard approximation to the nonlinear term in the RLW equation by investigating the effects of the modified equation on the single solitary wave solution (8). Warming and Hyett [43] investigate the stability of simple linear partial differential equations by investigating the modified partial differential equation. In a series of papers Villatoro and Ramos [4447] used the modified equation approach to analyze and improve the efficiency of an Euler forward difference method to solve linear and nonlinear differential equations. Junk and Yang [48] combine a modified equation and truncation error analysis in an asymptotic analysis of finite difference schemes. Rus and Villatoro [49] derive high-order finite difference 
schemes that preserve conserved quantities for RosenauHyman equation based on the corresponding modified equations.

The paper is divided up as follows: in Section 2 we derive and analyze the nonstandard finite difference schemes. The numerical schemes are simulated in Section 3. Concluding remarks are made in Section 4.

\section{Analysis of Modified Equation}

In this section we analyze the modified equation that we obtain from a finite difference approximation of the RLW equation (1). We define $u_{i}^{j}=u\left(x_{i}, t_{j}\right)$, where the spatial domain is discretized into $n+1$ points and $x_{i}=i \Delta x$, where $\Delta x$ is the spatial step length. The time $t_{j}$ is defined by $t_{j}=j \Delta t$, where $\Delta t$ is the time step length. Central difference approximations to the spatial derivatives are given by

$$
\begin{gathered}
\frac{\partial u}{\partial x} \approx \frac{u_{i+1}^{j}-u_{i-1}^{j}}{2 \Delta x}+\mathcal{O}\left(\Delta x^{2}\right), \\
\frac{\partial^{2} u}{\partial x^{2}} \approx \frac{u_{i+1}^{j}-2 u_{i}^{j}+u_{i-1}^{j}}{\Delta x^{2}}+\mathcal{O}\left(\Delta x^{2}\right) .
\end{gathered}
$$

A forward difference approximation to the time derivative is given by

$$
\frac{\partial u}{\partial t} \approx \frac{u_{i}^{j+1}-u_{i}^{j}}{\Delta t}+\mathcal{O}(\Delta t)
$$

We write the RLW equation (1) as

$$
\frac{\partial}{\partial t}\left(u-\frac{\partial^{2} u}{\partial x^{2}}\right)+\frac{\partial u}{\partial x}+\frac{1}{2} \frac{\partial u^{2}}{\partial x}=0
$$

Substituting (11), (12), and (13) with (14) we obtain an implicit numerical scheme given by

$$
\begin{gathered}
-\frac{1}{\Delta t}\left(u_{i+1}^{j+1}-u_{i+1}^{j}\right)+\left(\frac{\Delta x^{2}+2}{\Delta t}\right)\left(u_{i}^{j+1}-u_{i}^{j}\right) \\
-\frac{1}{\Delta t}\left(u_{i-1}^{j+1}-u_{i-1}^{j}\right)+\frac{\Delta x}{2}\left(u_{i+1}^{j}-u_{i-1}^{j}\right) \\
+\frac{\Delta x}{4}\left(\left(u_{i+1}^{j}\right)^{2}-\left(u_{i-1}^{j}\right)^{2}\right)=0,
\end{gathered}
$$

where we have approximated the partial derivative $\partial u / \partial x$ explicitly. To obtain the modified equation we substitute the Taylor series approximations

$$
\begin{aligned}
u_{i+1}^{j}= & u\left(x_{i+1}, t_{j}\right) \\
= & u\left(x_{i}, t_{j}\right)+\Delta x \frac{\partial u}{\partial x}\left(x_{i}, t_{j}\right) \\
& +\frac{\Delta x^{2}}{2 !} \frac{\partial^{2} u}{\partial x^{2}}\left(x_{i}, t_{j}\right)+O\left(\Delta x^{3}\right),
\end{aligned}
$$

$$
\begin{aligned}
u_{i-1}^{j}= & u\left(x_{i-1}, t_{j}\right) \\
= & u\left(x_{i}, t_{j}\right)-\Delta x \frac{\partial u}{\partial x}\left(x_{i}, t_{j}\right) \\
& +\frac{\Delta x^{2}}{2 !} \frac{\partial^{2} u}{\partial x^{2}}\left(x_{i}, t_{j}\right)-O\left(\Delta x^{3}\right),
\end{aligned}
$$

and similar approximations for $u_{i+1}^{j+1}, u_{i}^{j+1}$, and $u_{i-1}^{j+1}$ with (15) to obtain the partial differential equation

$$
\frac{\partial}{\partial t}\left(u-\frac{\partial^{2} u}{\partial x^{2}}\right)+\frac{\partial u}{\partial x}+\frac{1}{2} \frac{\partial u^{2}}{\partial x}+\frac{\Delta t}{2 \Delta x^{2}}\left(\frac{\partial^{2} u}{\partial t^{2}}-\frac{\partial^{4} u}{\partial t^{2} \partial x^{2}}\right)=0
$$

We let the modified equation be defined by $\Delta \epsilon$. Multiplying the modified equation $\Delta \epsilon$ by $\Delta t /\left(2 \Delta x^{2}\right)$ we obtain

$$
\frac{\Delta t \Delta \epsilon}{2 \Delta x^{2}}=\frac{\partial^{2} u}{\partial t^{2}}-\frac{\partial^{4} u}{\partial t^{2} \partial x^{2}} .
$$

To determine the effects of the modified equation (18) on the single solitary wave solution of the RLW equation (1) we substitute the single solitary wave solution (8) with (18) and plot $\Delta t \Delta \epsilon /\left(2 \Delta x^{2}\right)$ at different times in Figure 1 .

We improve the stability and accuracy of the numerical scheme (15) by approximating the derivative $\partial u / \partial x$ implicitly to obtain an implicit scheme to solve the RLW equation (14) given by

$$
\begin{aligned}
& -\frac{1}{\Delta t}\left(u_{i+1}^{j+1}-u_{i+1}^{j}\right)+\left(\frac{\Delta x^{2}+2}{\Delta t}\right)\left(u_{i}^{j+1}-u_{i}^{j}\right) \\
& -\frac{1}{\Delta t}\left(u_{i-1}^{j+1}-u_{i-1}^{j}\right)+\frac{\Delta x}{4}\left(u_{i+1}^{j+1}+u_{i+1}^{j}-u_{i-1}^{j+1}-u_{i-1}^{j}\right) \\
& +\frac{\Delta x}{4}\left(\left(u_{i+1}^{j}\right)^{2}-\left(u_{i-1}^{j}\right)^{2}\right)=0 .
\end{aligned}
$$

The corresponding modified equation is given by

$$
\frac{\Delta t \Delta \epsilon}{2 \Delta x^{2}}=\frac{\partial^{2} u}{\partial t^{2}}+\frac{\partial^{2} u}{\partial t \partial x}-\frac{\partial^{4} u}{\partial t^{2} \partial x^{2}} .
$$

Once again we determine the effects of the modified equation (20) on the single solitary wave solution of the RLW equation (1) by substituting the single solitary wave solution (8) with (20) and plotting the resulting function in Figure 1.

From the results indicated in Figure 1 we note that the impact of the modified equation on the single solitary wave solution of the RLW equation (1) is smaller for the modified equation (20) derived from the finite difference scheme (19) where the derivative $\partial u / \partial x$ is approximated implicitly than for (18) derived from the finite difference scheme (15) where the derivative $\partial u / \partial x$ is approximated explicitly. From this result we can conclude that the finite difference scheme (19) will yield better results than (15) when used to determine solutions to the RLW equation (1). We note that the curves in 


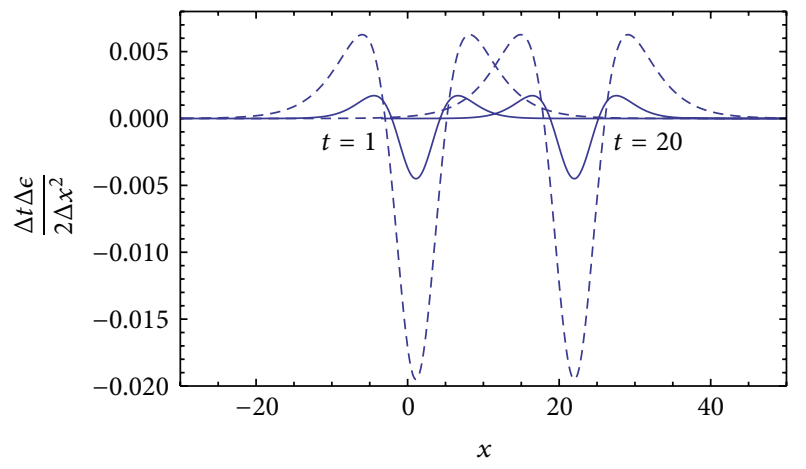

FIGURE 1: Plot comparing the modified equations obtained for explicit (18) (- - -) and implicit (20) (solid line) approximations to the derivative $\partial u / \partial x$ after substituting the single solitary wave solution to the RLW equation (8) with (18) and (20) taking $v=0.1$ and $x_{0}=0$.

Figure 1 propagate with the wave velocity of the single solitary wave solution (8) maintaining a constant wave amplitude.

We firstly consider the nonstandard approximation [41]

$$
\left(u_{i}^{j}\right)^{2} \longrightarrow 2\left(u_{i}^{j}\right)^{2}-u_{i}^{j+1} u_{i}^{j} .
$$

The implicit scheme (19) reduces to

$$
\begin{gathered}
-\frac{1}{\Delta t}\left(u_{i+1}^{j+1}-u_{i+1}^{j}\right)+\left(\frac{\Delta x^{2}+2}{\Delta t}\right)\left(u_{i}^{j+1}-u_{i}^{j}\right) \\
-\frac{1}{\Delta t}\left(u_{i-1}^{j+1}-u_{i-1}^{j}\right) \\
+\frac{\Delta x}{4}\left(u_{i+1}^{j+1}+u_{i+1}^{j}-u_{i-1}^{j+1}-u_{i-1}^{j}\right) \\
+\frac{\Delta x}{4}\left[\left(2\left(u_{i+1}^{j}\right)^{2}-u_{i+1}^{j+1} u_{i+1}^{j}\right)\right. \\
\left.\quad-\left(2\left(u_{i-1}^{j}\right)^{2}-u_{i-1}^{j+1} u_{i-1}^{j}\right)\right]=0,
\end{gathered}
$$

where the corresponding modified equation is given by

$$
\frac{\Delta t \Delta \epsilon}{2 \Delta x^{2}}=\frac{\partial^{2} u}{\partial t^{2}}-\frac{\partial u}{\partial t} \frac{\partial u}{\partial x}+(1-u) \frac{\partial^{2} u}{\partial t \partial x}-\frac{\partial^{4} u}{\partial t^{2} \partial x^{2}} .
$$

We next consider the nonstandard approximation [36]

$$
\left(u_{i-1}^{j}\right)^{2} \longrightarrow \bar{u}_{i-1}^{j} u_{i-1}^{j+1}, \quad\left(u_{i+1}^{j}\right)^{2} \longrightarrow \bar{u}_{i+1}^{j} u_{i+1}^{j+1},
$$

where

$$
\bar{u}_{i-1}^{j}=\frac{1}{2}\left(u_{i-1}^{j}+u_{i}^{j}\right), \quad \bar{u}_{i+1}^{j}=\frac{1}{2}\left(u_{i}^{j}+u_{i+1}^{j}\right) .
$$

The implicit scheme (19) reduces to

$$
\begin{aligned}
& -\frac{1}{\Delta t}\left(u_{i+1}^{j+1}-u_{i+1}^{j}\right)+\left(\frac{\Delta x^{2}+2}{\Delta t}\right)\left(u_{i}^{j+1}-u_{i}^{j}\right) \\
& -\frac{1}{\Delta t}\left(u_{i-1}^{j+1}-u_{i-1}^{j}\right)+\frac{\Delta x}{4}\left(u_{i+1}^{j+1}+u_{i+1}^{j}-u_{i-1}^{j+1}-u_{i-1}^{j}\right) \\
& +\frac{\Delta x}{8}\left[\left(u_{i}^{j}+u_{i+1}^{j}\right) u_{i+1}^{j+1}-\left(u_{i-1}^{j}+u_{i}^{j}\right) u_{i-1}^{j+1}\right]=0,
\end{aligned}
$$

where the corresponding modified equation is given by

$$
\frac{\Delta t \Delta \epsilon}{2 \Delta x^{2}}=\frac{\partial^{2} u}{\partial t^{2}}+\frac{1}{2} \frac{\partial u}{\partial t} \frac{\partial u}{\partial x}+(1+u) \frac{\partial^{2} u}{\partial t \partial x}-\frac{\partial^{4} u}{\partial t^{2} \partial x^{2}} .
$$

We substitute the single solitary wave solution (8) with (23) and (27) and plot the resulting functions in Figure 2 to determine the effects of the modified equations (23) and (27) on the single solitary wave solution of the RLW equation (1).

From the results indicated in Figure 2 we note that the impact of the modified equation (27) on the single solitary wave solution of the RLW equation (1) is smaller than that of the modified equation (23). We can therefore conclude that the nonstandard approximation (24) is a better approximation to use for the nonlinear term in the RLW equation than (21). We can also conclude that the finite difference scheme (26) will produce better results than (22) when used to determine numerical solutions to the RLW equation. In both cases the functions obtained after substituting the single solitary wave solution of the RLW equation (1) with the modified equations (23) and (27) propagate with the wave velocity of the single solitary wave solution.

We next consider the approximation [41]

$$
\left(u_{i}^{j}\right)^{2} \longrightarrow u_{i}^{j+1} u_{i}^{j}
$$

Substituting (28) with (19) we obtain the nonstandard numerical scheme

$$
\begin{aligned}
& -\frac{1}{\Delta t}\left(u_{i+1}^{j+1}-u_{i+1}^{j}\right)+\left(\frac{\Delta x^{2}+2}{\Delta t}\right)\left(u_{i}^{j+1}-u_{i}^{j}\right) \\
& -\frac{1}{\Delta t}\left(u_{i-1}^{j+1}-u_{i-1}^{j}\right)+\frac{\Delta x}{4}\left(u_{i+1}^{j+1}+u_{i+1}^{j}-u_{i-1}^{j+1}-u_{i-1}^{j}\right) \\
& \quad+\frac{\Delta x}{4}\left[u_{i+1}^{j+1} u_{i+1}^{j}-u_{i-1}^{j+1} u_{i-1}^{j}\right]=0 .
\end{aligned}
$$

The modified equation corresponding to (29) is given by

$$
\frac{\Delta t \Delta \epsilon}{\Delta x^{2}}=\frac{\partial}{\partial t}\left(\frac{\partial u}{\partial t}-\frac{\partial^{3} u}{\partial t \partial x^{2}}+\frac{\partial u}{\partial x}+u \frac{\partial u}{\partial x}\right)=0 .
$$




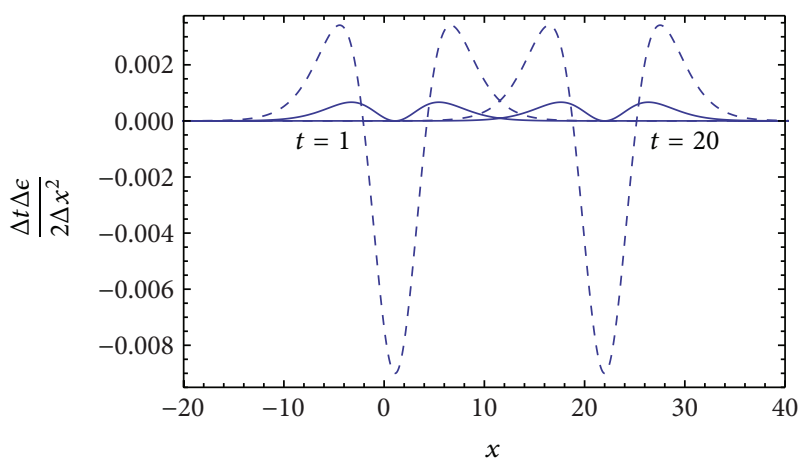

FIGURE 2: Plot comparing the behaviour of the modified equations obtained for the nonstandard approximation (21) $(---)$ and the nonstandard approximation (24) (solid line) to the nonlinear term in the RLW equation after substituting the single solitary wave solution to the RLW equation (8) with (23) and (27) taking $v=0.1$ and $x_{0}=0$.

We consider the next order in Taylor series expansions (16) to obtain the modified equation

$$
\frac{\Delta t^{2} \Delta \epsilon}{6 \Delta x^{4}}=\frac{\partial^{3} u}{\partial t^{3}}+\frac{3}{2} \frac{\partial^{2} u}{\partial t^{2}} \frac{\partial u}{\partial x}+\frac{3}{2} \frac{\partial^{3} u}{\partial t^{2} \partial x}(1+u)-\frac{\partial^{5} u}{\partial t^{3} \partial x^{2}} .
$$

We finally consider the approximation [39]

$$
\left(u_{i}^{j}\right)^{2} \longrightarrow \frac{1}{3}\left(u_{i-1}^{j}+u_{i}^{j}+u_{i+1}^{j}\right) u_{i}^{j+1} .
$$

The implicit scheme (19) reduces to

$$
\begin{aligned}
-\frac{1}{\Delta t}\left(u_{i+1}^{j+1}-u_{i+1}^{j}\right)+\left(\frac{\Delta x^{2}+2}{\Delta t}\right)\left(u_{i}^{j+1}-u_{i}^{j}\right) \\
-\frac{1}{\Delta t}\left(u_{i-1}^{j+1}-u_{i-1}^{j}\right)+\frac{\Delta x}{4}\left(u_{i+1}^{j+1}+u_{i+1}^{j}-u_{i-1}^{j+1}-u_{i-1}^{j}\right) \\
+\frac{\Delta x}{12}\left[\left(u_{i}^{j}+u_{i+1}^{j}+u_{i+2}^{j}\right) u_{i+1}^{j+1}\right. \\
\left.-\left(u_{i-2}^{j}+u_{i-1}^{j}+u_{i}^{j}\right) u_{i-1}^{j+1}\right]=0 .
\end{aligned}
$$

The corresponding modified equation is given by (30) and hence (31), respectively. We substitute the single solitary wave solution (8) with (31) and plot the resulting function in Figure 3.

From the results plotted in Figure 3 we observe that the second-order modified equation has the same order error as the first-order modified equations plotted in Figure 2. The solutions plotted in Figure 3 propagate with the wave velocity of the solitary wave solution.

Since the first-order modified equation (30) is identically satisfied we can conclude that the nonstandard finite difference schemes (29) and (33) are second-order accurate. Hence we can conclude that the nonstandard approximations (28) and (32) are the "best" approximations to use for the RLW equation. In addition, we obtain the CFL condition $\Delta t / \Delta x^{2} \ll 1$ for the stability of the implicit finite difference scheme (19) and $\Delta t^{2} / \Delta x^{4} \ll 1$ for the stability of the nonstandard finite difference schemes (29) and (33). In the next section we simulate the nonstandard finite difference schemes (29) and (33) to determine which scheme conserves (5)-(7) the "best."

\section{Simulation of Numerical Schemes}

For the first part of this section we take as the initial condition from the single solitary wave solution (8)

$$
u(x, 0)=\mathbf{u}^{0}=3 v \operatorname{sech}^{2}\left(k_{0} x\right)
$$

where $\mathbf{u}^{0}=\left[u_{0}^{0}, u_{1}^{0}, \ldots, u_{n}^{0}\right]$ and $k_{0}=(1 / 2) \sqrt{v /(1+v)}$. We choose $v=0.1$ and $x_{0}=0$ and divide the interval $x=$ $[-50,70]$ into $n+1$ equidistant points taking $\Delta t=0.0001$ and $n=5000$. We simulate the proposed nonstandard numerical schemes using MATHEMATICA on a $2.66 \mathrm{GHz}$ quad core Windows machine with 8.00 GB RAM.

We firstly consider the implicit scheme (19) that we write as

$$
\begin{gathered}
\left(-\frac{1}{\Delta t}+\frac{\Delta x}{4}\right) u_{i+1}^{j+1}+\left(\frac{\Delta x^{2}+2}{\Delta t}\right) u_{i}^{j+1}+\left(-\frac{1}{\Delta t}-\frac{\Delta x}{4}\right) u_{i-1}^{j+1} \\
=\left(-\frac{1}{\Delta t}-\frac{\Delta x}{4}\right) u_{i+1}^{j}+\left(\frac{\Delta x^{2}+2}{\Delta t}\right) u_{i}^{j} \\
\quad+\left(-\frac{1}{\Delta t}+\frac{\Delta x}{4}\right) u_{i-1}^{j}-\frac{\Delta x}{4}\left[\left(u_{i+1}^{j}\right)^{2}-\left(u_{i-1}^{j}\right)^{2}\right] .
\end{gathered}
$$

We then write the numerical scheme (35) as the linear system

$$
\mathbf{A} \mathbf{u}^{j+1}=\mathbf{d},
$$

where

$$
\mathbf{A}=\left[\begin{array}{ccccccccc}
1 & 0 & 0 & 0 & \cdots & 0 & 0 & 0 & 0 \\
a_{1} & b_{1} & c_{1} & 0 & \cdots & \vdots & \vdots & \vdots & \vdots \\
0 & a_{2} & b_{2} & c_{2} & \cdots & \vdots & \vdots & \vdots & \vdots \\
\vdots & \vdots & \vdots & \vdots & \vdots & \vdots & \vdots & \vdots & \vdots \\
\vdots & \vdots & \vdots & \vdots & \cdots & a_{n-2} & b_{n-2} & c_{n-2} & 0 \\
\vdots & \vdots & \vdots & \vdots & \cdots & 0 & a_{n-1} & b_{n-1} & c_{n-1} \\
0 & 0 & 0 & 0 & \cdots & 0 & 0 & 0 & 1
\end{array}\right],
$$




$$
\mathbf{d}=\left[\begin{array}{c}
0 \\
\left(-\frac{1}{\Delta t}-\frac{\Delta x}{4}\right) u_{2}^{j}+\left(\frac{\Delta x^{2}+2}{\Delta t}\right) u_{1}^{j}+\left(-\frac{1}{\Delta t}+\frac{\Delta x}{4}\right) u_{0}^{j}-\frac{\Delta x}{4}\left[\left(u_{2}^{j}\right)^{2}-\left(u_{0}^{j}\right)^{2}\right] \\
\left(-\frac{1}{\Delta t}-\frac{\Delta x}{4}\right) u_{3}^{j}+\left(\frac{\Delta x^{2}+2}{\Delta t}\right) u_{2}^{j}+\left(-\frac{1}{\Delta t}+\frac{\Delta x}{4}\right) u_{1}^{j}-\frac{\Delta x}{4}\left[\left(u_{3}^{j}\right)^{2}-\left(u_{1}^{j}\right)^{2}\right] \\
\vdots \\
\left(-\frac{1}{\Delta t}-\frac{\Delta x}{4}\right) u_{n-1}^{j}+\left(\frac{\Delta x^{2}+2}{\Delta t}\right) u_{n-2}^{j}+\left(-\frac{1}{\Delta t}+\frac{\Delta x}{4}\right) u_{n-3}^{j}-\frac{\Delta x}{4}\left[\left(u_{n-1}^{j}\right)^{2}-\left(u_{n-3}^{j}\right)^{2}\right] \\
\left(-\frac{1}{\Delta t}-\frac{\Delta x}{4}\right) u_{n}^{j}+\left(\frac{\Delta x^{2}+2}{\Delta t}\right) u_{n-1}^{j}+\left(-\frac{1}{\Delta t}+\frac{\Delta x}{4}\right) u_{n-2}^{j}-\frac{\Delta x}{4}\left[\left(u_{n}^{j}\right)^{2}-\left(u_{n-2}^{j}\right)^{2}\right] \\
0
\end{array}\right] .
$$

For the scheme (35)

$$
a_{i}=-\frac{1}{\Delta t}-\frac{\Delta x}{4}, \quad b_{i}=\frac{\Delta x^{2}+2}{\Delta t}, \quad c_{i}=-\frac{1}{\Delta t}+\frac{\Delta x}{4} .
$$

The boundary conditions (3) determine the first and last rows of $\mathbf{A}$ and $\mathbf{d}$. We use a Thomas algorithm [4] to solve the linear system of equations (36). We compare the single solitary wave solution (8) to the numerical solution obtained from the linear system (36) corresponding to the implicit scheme (35) using the $L_{2}$ and $L_{\infty}$ norms as defined in (10). We also evaluate the conserved quantities (5)-(7) using a trapezoidal quadrature rule. We tabulate our results in Table 3 . We find that the implicit scheme (35) does not preserve $I_{2}$ or $I_{3}$. At $t=20$ the $L_{2}$ and $L_{\infty}$ norms are significantly better than the $L_{2}$ and $L_{\infty}$ norms obtained by Avilez-Valente and SeabraSantos [6] and Dağ et al. [7] indicated in Tables 1 and 2, respectively.
We next consider the nonstandard numerical scheme (29). The nonstandard numerical scheme (29) can be written as

$$
\begin{gathered}
{\left[-\frac{1}{\Delta t}+\frac{\Delta x}{4}\left(1+u_{i+1}^{j}\right)\right] u_{i+1}^{j+1}+\left(\frac{\Delta x^{2}+2}{\Delta t}\right) u_{i}^{j+1}} \\
+\left[-\frac{1}{\Delta t}-\frac{\Delta x}{4}\left(1+u_{i-1}^{j}\right)\right] u_{i-1}^{j+1} \\
=\left(-\frac{1}{\Delta t}-\frac{\Delta x}{4}\right) u_{i+1}^{j}+\left(\frac{\Delta x^{2}+2}{\Delta t}\right) u_{i}^{j} \\
+\left(-\frac{1}{\Delta t}+\frac{\Delta x}{4}\right) u_{i-1}^{j} .
\end{gathered}
$$

We write the numerical scheme (40) as the system (36) where $\mathbf{A}$ is given by (37) and where

$$
\mathbf{d}=\left[\begin{array}{c}
\left(-\frac{1}{\Delta t}-\frac{\Delta x}{4}\right) u_{2}^{j}+\left(\frac{\Delta x^{2}+2}{\Delta t}\right) u_{1}^{j}+\left(-\frac{1}{\Delta t}+\frac{\Delta x}{4}\right) u_{0}^{j} \\
\left(-\frac{1}{\Delta t}-\frac{\Delta x}{4}\right) u_{3}^{j}+\left(\frac{\Delta x^{2}+2}{\Delta t}\right) u_{2}^{j}+\left(-\frac{1}{\Delta t}+\frac{\Delta x}{4}\right) u_{1}^{j} \\
\vdots \\
\left(-\frac{1}{\Delta t}-\frac{\Delta x}{4}\right) u_{n-1}^{j}+\left(\frac{\Delta x^{2}+2}{\Delta t}\right) u_{n-2}^{j}+\left(-\frac{1}{\Delta t}+\frac{\Delta x}{4}\right) u_{n-3}^{j} \\
\left(-\frac{1}{\Delta t}-\frac{\Delta x}{4}\right) u_{n}^{j}+\left(\frac{\Delta x^{2}+2}{\Delta t}\right) u_{n-1}^{j}+\left(-\frac{1}{\Delta t}+\frac{\Delta x}{4}\right) u_{n-2}^{j} \\
0
\end{array}\right]
$$

such that

$$
\begin{gathered}
a_{i}=-\frac{1}{\Delta t}-\frac{\Delta x}{4}\left(1+u_{i-1}^{j}\right), \quad b_{i}=\frac{\Delta x^{2}+2}{\Delta t}, \\
c_{i}=-\frac{1}{\Delta t}+\frac{\Delta x}{4}\left(1+u_{i+1}^{j}\right) .
\end{gathered}
$$

We tabulate our results in Table 4 . We find that the nonstandard numerical scheme (40) shows no deviation in the evaluation of the conserved quantities (5)-(7). Also, the $L_{2}$ and $L_{\infty}$ norms are once again significantly better than the $L_{2}$ and $L_{\infty}$ norms obtained by Avilez-Valente and Seabra-Santos [6] and Dağ et al. [7] indicated in Tables 1 and 2, respectively. 
TABLE 3: Table comparing values obtained from the implicit scheme (35) to the single solitary wave solution (8) for $x_{0}=0$ and $v=0.1$, for $x \in[-50,70]$, and taking $n=5000$ and $\Delta t=0.0001$.

\begin{tabular}{lccccc}
\hline Time & $I_{1}$ & $I_{2}$ & $I_{3}$ & $L_{2} \times 10^{3}$ & $L_{\infty} \times 10^{3}$ \\
\hline 0 & 3.97995 & 0.810462 & 2.57901 & 0.00000 & 0.00000 \\
4 & 3.97995 & 0.810463 & 2.57901 & 0.0044822 & 0.00190039 \\
8 & 3.97995 & 0.810464 & 2.57901 & 0.00884828 & 0.00377625 \\
12 & 3.97995 & 0.810466 & 2.57902 & 0.0130448 & 0.00550027 \\
16 & 3.97995 & 0.810467 & 2.57902 & 0.0170392 & 0.00705772 \\
20 & 3.97995 & 0.810468 & 2.57903 & 0.0208237 & 0.00846861 \\
\hline
\end{tabular}

TABLE 4: Table comparing values obtained from the nonstandard numerical scheme (40) to the single solitary wave solution $(8)$ for $x_{0}=0$ and $v=0.1$, for $x \in[-50,70]$, and taking $n=5000$ and $\Delta t=0.0001$.

\begin{tabular}{lccccc}
\hline Time & $I_{1}$ & $I_{2}$ & $I_{3}$ & $L_{2} \times 10^{3}$ & $L_{\infty} \times 10^{3}$ \\
\hline 0 & 3.97995 & 0.810462 & 2.57901 & 0.00000 & 0.00000 \\
4 & 3.97995 & 0.810462 & 2.57901 & 0.00424465 & 0.00167944 \\
8 & 3.97995 & 0.810462 & 2.57901 & 0.00839433 & 0.00337615 \\
12 & 3.97995 & 0.810462 & 2.57901 & 0.012402 & 0.00495651 \\
16 & 3.97995 & 0.810462 & 2.57901 & 0.0162382 & 0.00639525 \\
20 & 3.97995 & 0.810462 & 2.57901 & 0.0198962 & 0.00770612 \\
\hline
\end{tabular}

We finally consider the nonstandard numerical scheme (33). The nonstandard numerical scheme (33) can be written as

$$
\begin{aligned}
& {\left[-\frac{1}{\Delta t}+\frac{\Delta x}{4}+\frac{\Delta x}{12}\left(u_{i}^{j}+u_{i+1}^{j}+u_{i+2}^{j}\right)\right] u_{i+1}^{j+1}} \\
& \quad+\left(\frac{\Delta x^{2}+2}{\Delta t}\right) u_{i}^{j+1} \\
& \quad+\left[-\frac{1}{\Delta t}-\frac{\Delta x}{4}-\frac{\Delta x}{12}\left(u_{i-2}^{j}+u_{i-1}^{j}+u_{i}^{j}\right)\right] u_{i-1}^{j+1} \\
& =\left(-\frac{1}{\Delta t}-\frac{\Delta x}{4}\right) u_{i+1}^{j}+\left(\frac{\Delta x^{2}+2}{\Delta t}\right) u_{i}^{j} \\
& \quad+\left(-\frac{1}{\Delta t}+\frac{\Delta x}{4}\right) u_{i-1}^{j} .
\end{aligned}
$$

We write (43) as the linear system (36) where $\mathbf{A}$ and $\mathbf{d}$ are given by (37) and (41) such that

$$
\begin{gathered}
a_{1}=-\frac{1}{\Delta t}-\frac{\Delta x}{4}-\frac{\Delta x}{12}\left(2 u_{1}^{j}+u_{0}^{j}\right), \\
a_{i}=-\frac{1}{\Delta t}-\frac{\Delta x}{4}-\frac{\Delta x}{12}\left(u_{i-2}^{j}+u_{i-1}^{j}+u_{i}^{j}\right), \quad i=2, \ldots, n-1, \\
c_{n-1}=-\frac{1}{\Delta t}+\frac{\Delta x}{4}+\frac{\Delta x}{12}\left(2 u_{n-1}^{j}+u_{n}^{j}\right), \\
c_{i}=-\frac{1}{\Delta t}+\frac{\Delta x}{4}+\frac{\Delta x}{12}\left(u_{i}^{j}+u_{i+1}^{j}+u_{i+2}^{j}\right), \quad i=1, \ldots, n-2, \\
b_{i}=\frac{\Delta x^{2}+2}{\Delta t}, \quad i=1, \ldots, n-1 .
\end{gathered}
$$

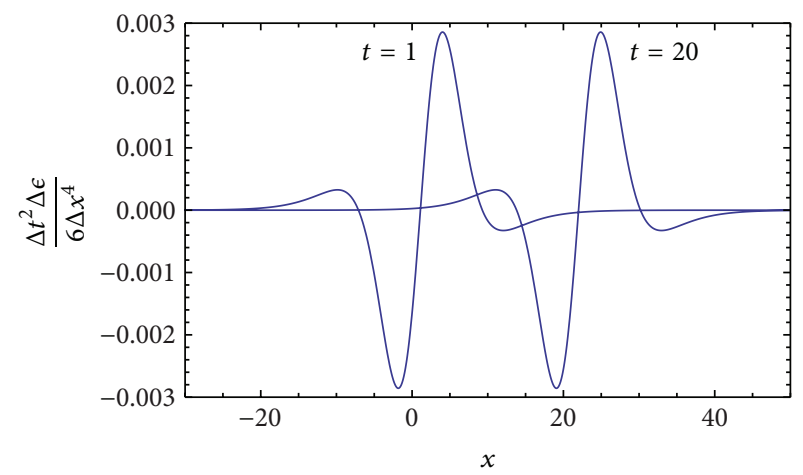

FIGURE 3: Plot showing the behaviour of the second-order modified equation (31) after substituting the single solitary wave solution to the RLW equation (8) with (31) taking $v=0.1$ and $x_{0}=0$.

The unknown values of $u$ in $a_{1}$ and $c_{n-1}$ are determined from Neumann boundary conditions obtained from the central difference approximation (11)

$$
\frac{u_{i+1}^{j}-u_{i-1}^{j}}{2 \Delta x}=0 .
$$

Evaluating (45) at $i=0$ and $i=n$ we get that $u_{-1}^{j}=u_{1}^{j}$ and $u_{n+1}^{j}=u_{n-1}^{j}$. We tabulate the results obtained from the numerical scheme (43) in Table 5. We find that the nonstandard numerical scheme (43) conserves $I_{1}, I_{2}$, and $I_{3}$ obtained from (5)-(7). Once again the $L_{2}$ and $L_{\infty}$ norms at $t=20$ are significantly better than the $L_{2}$ and $L_{\infty}$ norms obtained by Avilez-Valente and Seabra-Santos [6] and Dağ et al. [7] indicated in Tables 1 and 2, respectively.

In Figure 4 we plot the time evolution of the $L_{2}$ and $L_{\infty}$ norms for the implicit scheme (35) and the nonstandard numerical schemes (40) and (43). We note from Figure 4 
TABLE 5: Table comparing values obtained from the nonstandard numerical scheme (43) to the single solitary wave solution $(8)$ for $x_{0}=0$ and $v=0.1$, for $x \in[-50,70]$, and taking $n=5000$ and $\Delta t=0.0001$.

\begin{tabular}{lccccc}
\hline Time & $I_{1}$ & $I_{2}$ & $I_{3}$ & $L_{2} \times 10^{3}$ & $L_{\infty} \times 10^{3}$ \\
\hline 0 & 3.97995 & 0.810462 & 2.57901 & 0.00000 & 0.00000 \\
4 & 3.97995 & 0.810462 & 2.57901 & 0.00514014 & 0.00207598 \\
8 & 3.97995 & 0.810462 & 2.57901 & 0.0101538 & 0.00416179 \\
12 & 3.97995 & 0.810462 & 2.57901 & 0.0149734 & 0.0060774 \\
16 & 3.97995 & 0.810462 & 2.57901 & 0.019566 & 0.00780266 \\
20 & 3.97995 & 0.810462 & 2.57901 & 0.023929 & 0.00936437 \\
\hline
\end{tabular}
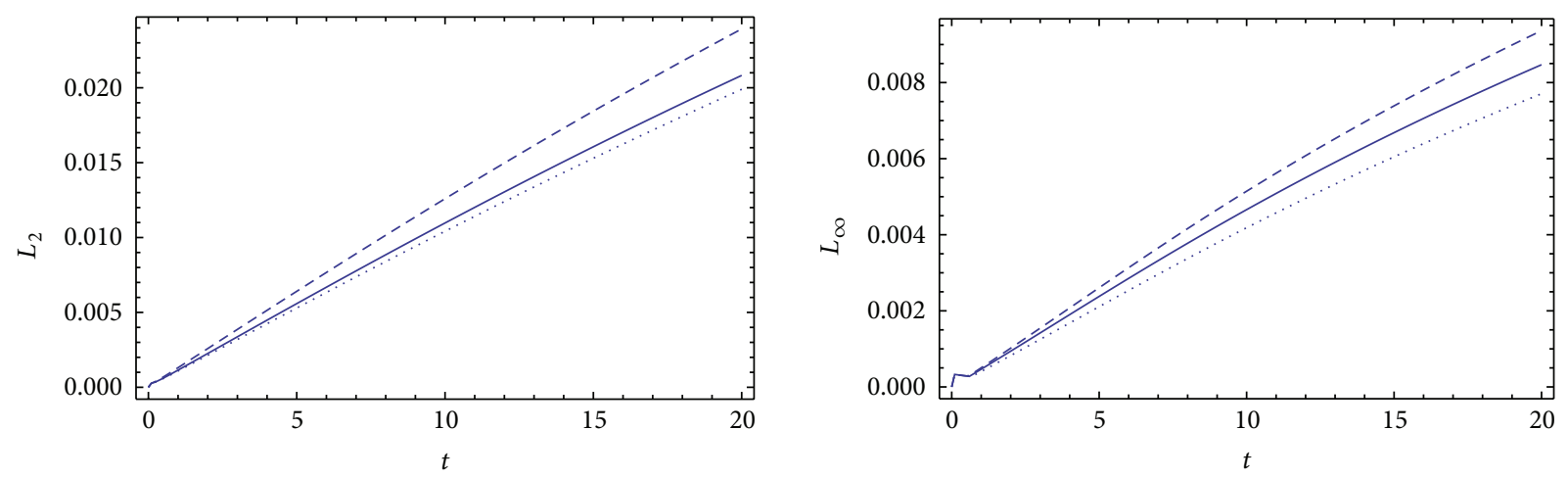

Figure 4: Plot showing the time evolution of the $L_{2}$ and $L_{\infty}$ norms for the implicit scheme (35) (solid line), the nonstandard numerical

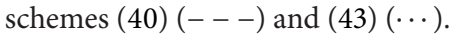

that the implicit scheme (35) performs better than the nonstandard numerical scheme (43) in terms of the growth in the error. The nonstandard numerical (40) is the best performer in terms of the growth in the error. From the work of Araújo and Durán [31] we can conclude that because the growth in the errors is linear and the nonstandard numerical schemes preserve the invariants (5)-(7) the schemes (40) and (43) are conservative.

In this section we have also shown that the implicit scheme (35), with no nonstandard approximation to the nonlinear term, produces results that do not preserve the invariants (5)-(7), even though at $t=20$ the $L_{2}$ and $L_{\infty}$ norms are significantly better than the results obtained by Avilez-Valente and Seabra-Santos [6] and Dağ et al. [7]. The two nonstandard numerical schemes (40) and (43) preserve the invariants (5)-(7) and produce $L_{2}$ and $L_{\infty}$ norms at $t=20$ that are significantly better than the results of Avilez-Valente and Seabra-Santos [6] and Dağ et al. [7].

From the results simulated above and captured in Tables 3 , 4 , and 5 we note that for the initial single solitary wave (34) the implicit scheme (35) and the nonstandard numerical schemes (40) and (43) preserve the conserved quantities (5)(7).

In order to test how effectively the implicit scheme (35) and the nonstandard numerical schemes (40) and (43) solve the RLWE equation, we consider the difference $u(x, t+\Delta t)-$ $u(x, t)$ between two solutions. We define

$$
\Phi_{0,1,2}=u\left(\mathbf{x}, t_{j}+\Delta t\right)-u\left(\mathbf{x}, t_{j}\right)=\mathbf{u}^{j+1}-\mathbf{u}^{j}
$$

where the subscripts $0,1,2$ correspond to the implicit scheme (35) and the nonstandard numerical schemes (40) and (43), respectively. From (36) we have that $\mathbf{A u}^{j+1}=\mathbf{d}$. Equation (36) gives $\mathbf{u}^{j+1}=\mathbf{A}^{-1} \mathbf{d}$ leading us to write (46) as

$$
\bar{\Phi}_{0,1,2}=A \Phi_{0,1,2}=\mathbf{d}-\mathbf{A u}^{j} .
$$

The $i$ th row of the vector $\bar{\Phi}_{0,1,2}$ is given by

$$
\left[\bar{\Phi}_{0,1,2}\right]_{i}^{j}=\mathbf{d}_{i}^{j}-\left(a_{i} u_{i-1}^{j}+b_{i} u_{i}^{j}+c_{i} u_{i+1}^{j}\right) .
$$

For the implicit scheme (35) where (38) holds for $\mathbf{d}$ and (39) holds for $a_{i}, b_{i}$, and $c_{i}$ we find that

$$
\begin{gathered}
{\left[\bar{\Phi}_{0}\right]_{i}^{j}=\frac{\Delta x}{2}\left[u_{i-1}^{j}\left(1+\frac{1}{2} u_{i-1}^{j}\right)-u_{i+1}^{j}\left(1+\frac{1}{2} u_{i+1}^{j}\right)\right],} \\
{\left[\bar{\Phi}_{0}\right]_{0}^{j}=0, \quad\left[\bar{\Phi}_{0}\right]_{n}^{j}=0 .}
\end{gathered}
$$

For the nonstandard numerical scheme (40) the vector d is given by (41) and $a_{i}, b_{i}$, and $c_{i}$ are given by (42). Substitution with (47) yields

$$
\begin{gathered}
{\left[\bar{\Phi}_{1}\right]_{i}^{j}=\frac{\Delta x}{2}\left[u_{i-1}^{j}\left(1+\frac{1}{2} u_{i-1}^{j}\right)-u_{i+1}^{j}\left(1+\frac{1}{2} u_{i+1}^{j}\right)\right],} \\
{\left[\bar{\Phi}_{1}\right]_{0}^{j}=0, \quad\left[\bar{\Phi}_{1}\right]_{n}^{j}=0 .}
\end{gathered}
$$

This is the same as the expression (49). 

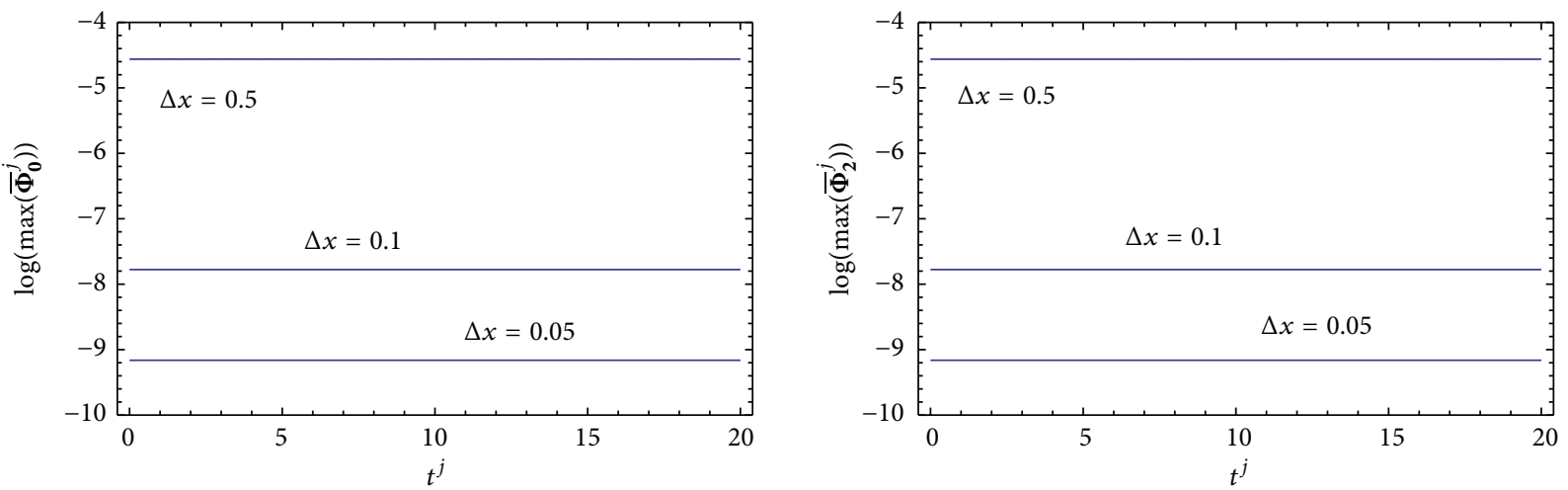

FIGURE 5: Figures showing the quantities $\log \left(\max \overline{\boldsymbol{\Phi}}_{0}^{j}\right)$ and $\log \left(\max \overline{\boldsymbol{\Phi}}_{2}^{j}\right)$ for each $t_{j}$, where $t_{j} \in[0,20], x_{0}=0$, and $v=0.1$ for $x \in[-50,70]$ for the step lengths $\Delta x=0.5, \Delta x=0.1$, and $\Delta x=0.05$.

For the nonstandard numerical scheme (43) the vector d is given by (41) and $a_{i}, b_{i}$, and $c_{i}$ are given by (44). Substitution with (47) yields

$$
\begin{gathered}
{\left[\bar{\Phi}_{2}\right]_{i}^{j}=\frac{\Delta x}{2}\left[u_{i-1}^{j}+\frac{1}{6}\left(u_{i-2}^{j}+u_{i-1}^{j}+u_{i}^{j}\right) u_{i-1}^{j}\right.} \\
\left.-u_{i+1}^{j}-\frac{1}{6}\left(u_{i}^{j}+u_{i+1}^{j}+u_{i+2}^{j}\right) u_{i+1}^{j}\right], \\
{\left[\bar{\Phi}_{2}\right]_{1}^{j}=\frac{\Delta x}{2}\left[u_{0}^{j}+\frac{1}{6}\left(u_{0}^{j}+2 u_{1}^{j}\right) u_{0}^{j}\right.} \\
\left.-u_{2}^{j}-\frac{1}{6}\left(u_{1}^{j}+u_{2}^{j}+u_{3}^{j}\right) u_{2}^{j}\right], \\
{\left[\bar{\Phi}_{2}\right]_{n-1}^{j}=\frac{\Delta x}{2}\left[u_{n-2}^{j}+\frac{1}{6}\left(u_{n-3}^{j}+u_{n-2}^{j}+u_{n-1}^{j}\right) u_{n-2}^{j}\right.} \\
\left.-u_{n}^{j}-\frac{1}{6}\left(2 u_{n-1}^{j}+u_{n}^{j}\right) u_{n}^{j}\right], \\
{\left[\bar{\Phi}_{2}\right]_{0}^{j}=0, \quad\left[\bar{\Phi}_{2}\right]_{n}^{j}=0 .}
\end{gathered}
$$

Conditions (50), (52), and (56) occur as a result of the Dirichlet boundary conditions.

To test how accurately the numerical schemes solve the RLWE we substitute in the solution to the RLWE equation given by (8) that we write as

$$
u\left(x_{i}, t_{j}\right)=u_{i}^{j}=3 v \operatorname{sech}^{2}\left(k_{0} x_{i}+k_{1} t_{j}+x_{0}\right),
$$

where

$$
k_{0}=\frac{1}{2} \sqrt{\frac{v}{1+v}}, \quad k_{1}=-\frac{1}{2} \sqrt{v(1+v)},
$$

with the respective differences (49) and (53)-(55). We plot $\log \left(\max \overline{\boldsymbol{\Phi}}_{0}^{j}\right)$ and $\log \left(\max \overline{\boldsymbol{\Phi}}_{2}^{j}\right)$ for each $t_{j}$ for different step lengths $\Delta x$ in Figure 5. We take $x_{0}=0$ and $v=0.1$ for $x \in$ $[-50,70]$. In Figure 6 we plot the difference $\log \left(\max \overline{\boldsymbol{\Phi}}_{0}^{j}\right)-$ $\log \left(\max \overline{\boldsymbol{\Phi}}_{2}^{j}\right)$.

It is clear from the expressions for $\left[\bar{\Phi}_{0,1,2}\right]_{i}^{j}$ that the amplitude of the oscillations in the trigonometric functions

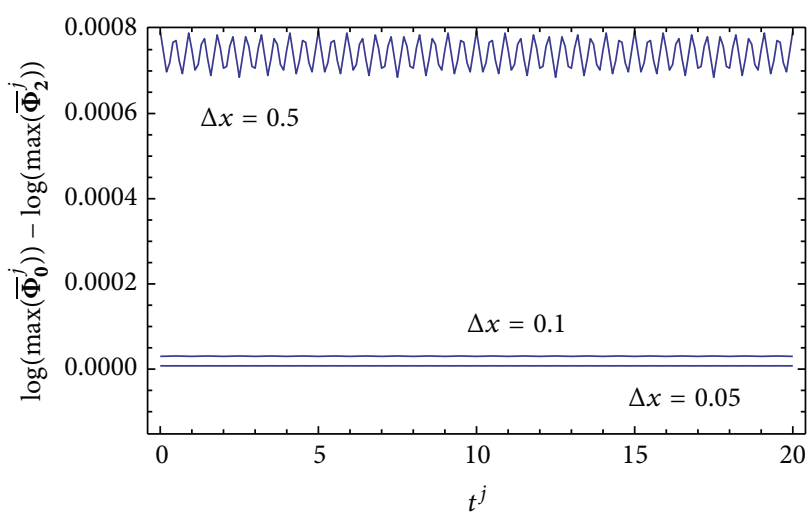

FIGURE 6: Figures showing the quantities $\log \left(\max \overline{\boldsymbol{\Phi}}_{0}^{j}\right)-\log \left(\max \overline{\boldsymbol{\Phi}}_{2}^{j}\right)$ for each $t_{j}$, where $t_{j} \in[0,20], x_{0}=0$, and $v=0.1$ for $x \in[-50,70]$ for the step lengths $\Delta x=0.5, \Delta x=0.1$, and $\Delta x=0.05$.

obtained by substituting (57) with (49)-(55), respectively, is proportional to the step length $\Delta x$. This is seen in the results plotted in Figure 5 but also more clearly in the results plotted in Figure 6. We note that as $\Delta x \rightarrow 0$ the maximum values of the differences plotted in both Figures 5 and 6 tend to zero.

We can therefore conclude that for the implicit numerical scheme (35) and the nonstandard numerical schemes (40) and (43) the difference given by (46) is oscillatory where the maximum height of the oscillation is proportional to the spatial step length. We can also conclude that the difference (46) tends to zero as $\Delta x \rightarrow 0$. We can therefore conclude that the first integral (5) that can be written as (46) on differentiating (5) with respect to time is satisfied by the implicit numerical scheme (35) and the nonstandard numerical schemes (40) and (43).

3.1. Undular Bore. We next apply the implicit scheme (35) and the nonstandard finite difference schemes (40) and (43) to model the evolution of an undular bore where the initial profile is given by

$$
u(x, 0)=\frac{1}{2} u_{0}\left(1-\tanh \left(\frac{x-x_{0}}{\alpha}\right)\right) .
$$



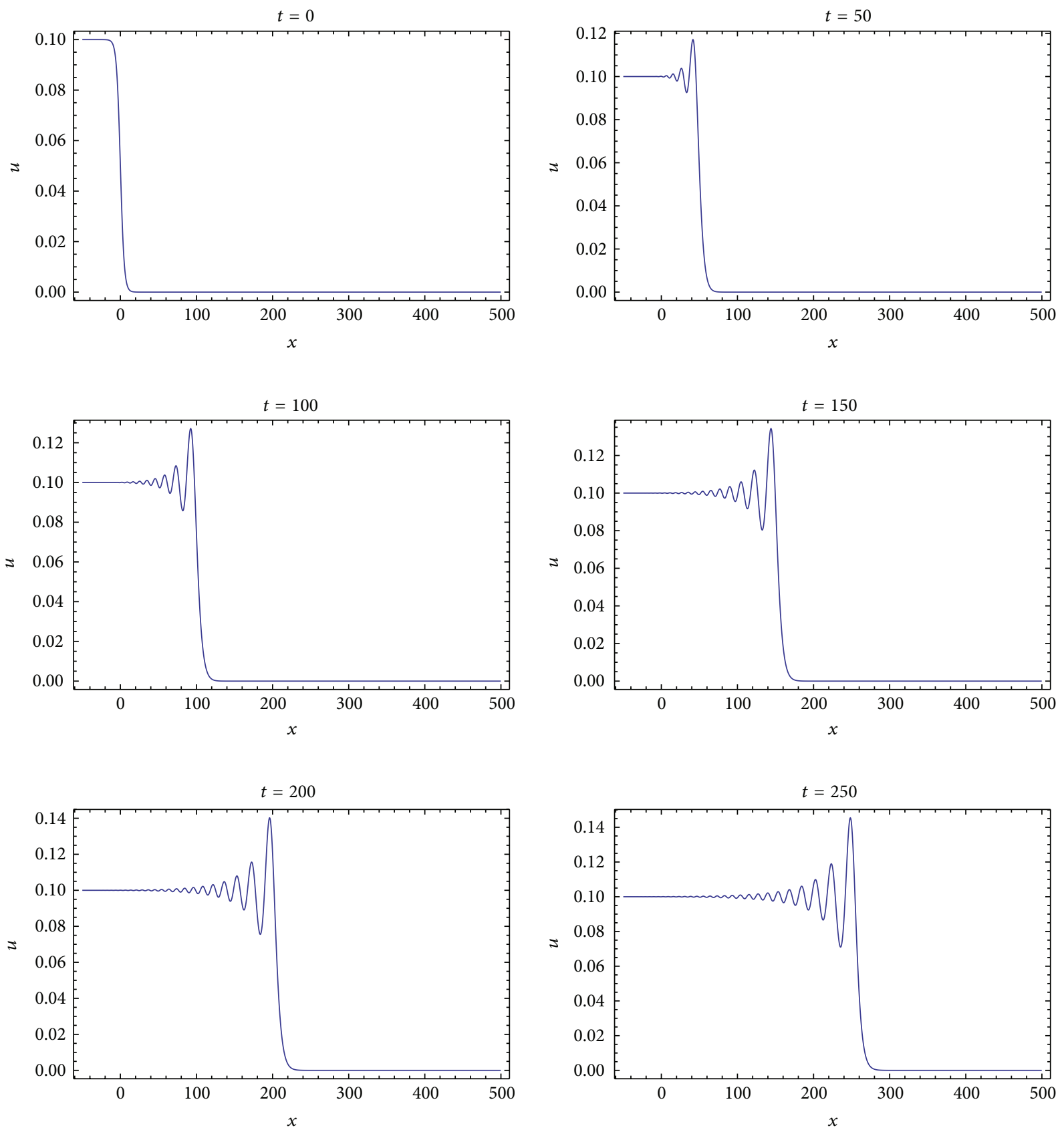

FIGURE 7: Evolution of initial undular bore (59), where $u_{0}=0.1, x_{0}=0$, and $\alpha=5$ for $\Delta t=0.001$ and $n=2500$ on the interval $x \in[-50,500]$ using the nonstandard finite difference scheme (40).

When simulating the development of an undular bore, we set the first row of the vector $\mathbf{d}$ in (38) and (41) to $d_{0}=u\left(x_{0}, 0\right)=$ $u_{0}$. We simulate the evolution of the undular bore using the implicit scheme (35) and the nonstandard finite difference schemes (40) and (43). We tabulate the maximum value of $u$ at the times indicated in Tables 6 and 7 for $\alpha=5$ and $\alpha=2$. From the results indicated in Tables 6 and 7 we note that the maximum values of $u$ for all three schemes compare well for $\alpha=5$ and $\alpha=2$. We plot a simulation of the evolution of the undular bore using the nonstandard finite difference scheme (40) in Figures 7 and 8 as this scheme produced the best results as tabulated in Table 4 . We choose $u_{0}=0.1, x_{0}=0$, and $\alpha=5$ for the curves in Figure 7 and $\alpha=2$ for the curves in Figure 8.

We note that the nonstandard numerical scheme (40) has captured the development of the undular bore without the formation of numerical instabilities. Our results are comparable with those obtained in Figures 9 and 11 of the paper by Avilez-Valente and Seabra-Santos [6]. We note that the front of the bore and maximum amplitude of the bore in Figures 7 and 8 are the same as those in Figures 9 and 11 of Avilez-Valente and Seabra-Santos [6], respectively. 

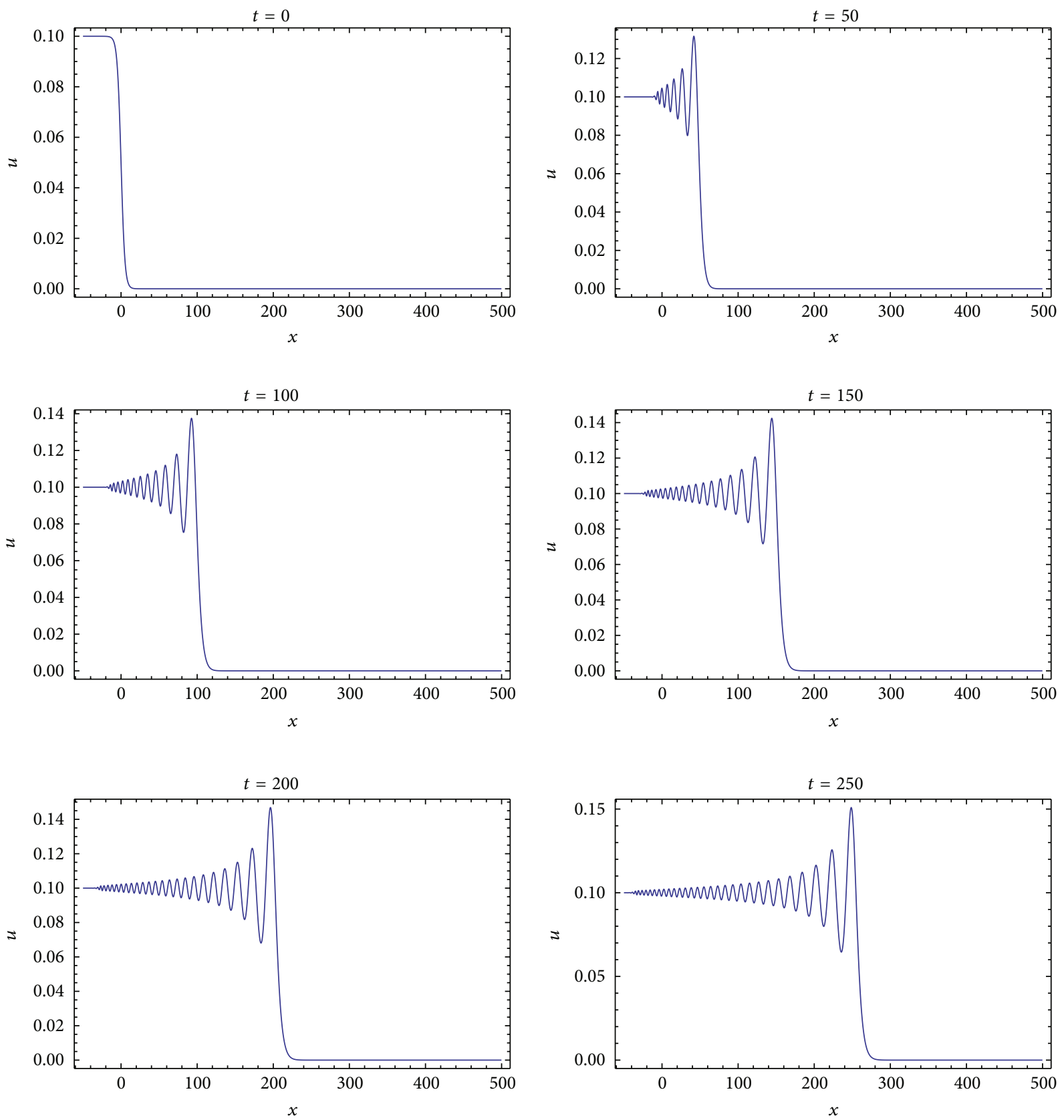

FIGURE 8: Evolution of initial undular bore (59), where $u_{0}=0.1, x_{0}=0$, and $\alpha=2$ for $\Delta t=0.001$ and $n=2500$ on the interval $x \in[-50$, 500] using the nonstandard finite difference scheme (40).

TABLE 6: Table showing max $u(x, t)$ for each of the schemes, where $\alpha=5$.

\begin{tabular}{lccc}
\hline Time & $(35)$ & $(40)$ & $(43)$ \\
\hline 50 & 0.117140 & 0.117136 & 0.117139 \\
100 & 0.127146 & 0.127139 & 0.127140 \\
150 & 0.134316 & 0.134306 & 0.134306 \\
200 & 0.140250 & 0.140236 & 0.140233 \\
250 & 0.145455 & 0.145438 & 0.145436 \\
\hline
\end{tabular}

TABLE 7: Table showing $\max u(x, t)$ for each of the schemes where $\alpha=2$.

\begin{tabular}{lccc}
\hline Time & $(35)$ & $(40)$ & $(43)$ \\
\hline 50 & 0.131598 & 0.131592 & 0.131591 \\
100 & 0.137537 & 0.137527 & 0.137526 \\
150 & 0.142442 & 0.142429 & 0.142427 \\
200 & 0.146848 & 0.146831 & 0.146827 \\
250 & 0.150901 & 0.150881 & 0.150879 \\
\hline
\end{tabular}




\section{Concluding Remarks}

In this paper we have shown the following:

(i) that the modified equation can be used as a criterion for selecting the "best" nonstandard finite difference scheme for solving the RLW equation (1);

(ii) that the nonstandard numerical scheme we have chosen does indeed perform well by preserving the three conserved quantities (5)-(7) and capturing the single solitary wave solution (8) accurately;

(iii) that the nonstandard finite difference scheme captures the development of an undular bore for both steep and shallow initial profiles.

The implicit nature of the nonstandard numerical schemes (40) and (43) derived in this paper means that at each time step the system matrix $A$ in (36) is recalculated and the system resolved. The numerical results obtained in this paper have been compared to the results obtained by Avilez-Valente and Seabra-Santos [6] who implemented a Petrov-Galerkin scheme and Dağ et al. [7] who implemented a splitted quadratic B-spline collocation scheme. In the Petrov-Galerkin scheme implemented by Avilez-Valente and Seabra-Santos [6] finite elements are used in both the space and time dimensions. The time-integration is performed by use of a predictor-corrector method. Avilez-Valente and Seabra-Santos [6] summarize the computational efficiency of their method by indicating that their FEM method leads to a tridiagonal system of $n+1$ equations, where $n$ is the number of spatial finite elements. The system matrix remains unchanged during the computation but three inner iterations are required at each time step as part of the predictorcorrector method. Avilez-Valente and Seabra-Santos [6] also comment on the fact that the splitted quadratic B-spline collocation scheme implemented by Dağ et al. [7] leads to a pentadiagonal system of $2 n+4$ equations. At each time step there are multiple inner iterations that need to be evaluated depending on the time-integration scheme that has been chosen. Unlike the Petrov-Galerkin method the system matrix must be reassembled after each time step. This makes the splitted quadratic B-spline collocation scheme implemented by Dağ et al. [7] computationally more expensive than the Petrov-Galerkin scheme implemented by AvilezValente and Seabra-Santos [6]. The nonstandard numerical schemes derived in this paper are easy to implement, highly accurate, and computationally a lot cheaper than either the Petrov-Galerkin numerical scheme or the splitted quadratic B-spline collocation scheme.

\section{Conflict of Interests}

The author declares that there is no conflict of interests regarding the publication of this paper.

\section{Acknowledgment}

E. Momoniat acknowledges support from the National Research Foundation of South Africa.

\section{References}

[1] D. H. Peregrine, "Calculations of the development of an undular Bore," Journal of Fluid Mechanics, vol. 25, no. 2, pp. 321-330, 1966.

[2] T. B. Benjamin, J. L. Bona, and J. J. Mahony, "Model equations for long waves in non-linear dispersive systems," Philosophical Transactions of the Royal Society of London A, vol. 272, no. 1220, pp. 47-78, 1972.

[3] D. J. Korteweg and G. de Vries, "On the change of form of long waves advancing in a rectangular canal, and on a new type of long stationary waves," Philosophical Magazine, vol. 39, pp. 422443, 1895.

[4] S. D. Conte and C. de Boor, Elementary Numerical Analysis, McGraw-Hill, New York, NY, USA, 1972.

[5] P. J. Olver, "Euler operators and conservation laws of the BBM equation," Mathematical Proceedings of the Cambridge Philosophical Society, vol. 85, no. 1, pp. 143-160, 1979.

[6] P. Avilez-Valente and F. J. Seabra-Santos, "A Petrov-Galerkin finite element scheme for the regularized long wave equation," Computational Mechanics, vol. 34, no. 4, pp. 256-270, 2004.

[7] I. Dağ, A. Doğan, and B. Saka, "B-spline collocation methods for numerical solutions of the RLW equation," International Journal of Computer Mathematics, vol. 80, no. 6, pp. 743-757, 2003.

[8] J. C. Eilbeck and G. R. McGuire, "Numerical study of the regularized long-wave equation I: numerical methods," Journal of Computational Physics, vol. 19, no. 1, pp. 43-57, 1975.

[9] J. C. Eilbeck and G. R. McGuire, "Numerical study of the regularized long-wave equation. II: Interaction of solitary waves," Journal of Computational Physics, vol. 23, no. 1, pp. 63-73, 1977.

[10] S. Kutluay and A. Esen, "A finite difference solution of the regularized long-wave equation," Mathematical Problems in Engineering, vol. 2006, pp. 1-14, 2006.

[11] P. C. Jain, R. Shankar, and T. V. Singh, "Numerical solution of regularized long-wave equation," Communications in Numerical Methods in Engineering, vol. 9, no. 7, pp. 579-586, 1993.

[12] J. L. Bona, W. G. Pritchard, and L. R. Scott, "Numerical schemes for a model for nonlinear dispersive waves," Journal of Computational Physics, vol. 60, no. 2, pp. 167-186, 1985.

[13] M. E. Alexander and J. L. Morris, "Galerkin methods applied to some model equations for non-linear dispersive waves," Journal of Computational Physics, vol. 30, no. 3, pp. 428-451, 1979.

[14] I. Dağ, "Least-squares quadratic B-spline finite element method for the regularised long wave equation," Computer Methods in Applied Mechanics and Engineering, vol. 182, no. 1-2, pp. 205215, 2000.

[15] L. R. T. Gardner, "Solitary waves of the regularised long-wave equation," Journal of Computational Physics, vol. 91, no. 2, pp. 441-459, 1990.

[16] L. R. T. Gardner, G. A. Gardner, F. A. Ayoub, and N. K. Amein, "Modelling an undular bore with B-splines," Computer Methods in Applied Mechanics and Engineering, vol. 147, no. 1-2, pp. 147152, 1997.

[17] L. R. T. Gardner, G. A. Gardner, and I. Dag, "A B-spline finite element method for the regularized long wave equation," Communications in Numerical Methods in Engineering, vol. 11, no. 1, pp. 59-68, 1995.

[18] L. R. T. Gardner, G. A. Gardner, and A. Dogan, "A least-squares finite element scheme for the RLW equation," Communications in Numerical Methods in Engineering, vol. 12, no. 11, pp. 795-804, 1996. 
[19] J. M. Sanz-Serna and I. Christie, "Petrov-Galerkin methods for nonlinear dispersive waves," Journal of Computational Physics, vol. 39, no. 1, pp. 94-102, 1981.

[20] Z. Luo and R. Liu, "Mixed finite element analysis and numerical solitary solution for the RLW equation," SIAM Journal on Numerical Analysis, vol. 36, no. 1, pp. 89-104, 1999.

[21] G. Ben-yu and V. S. Manoranjan, "A spectral method for solving the RLW equation," IMA Journal of Numerical Analysis, vol. 5, no. 3, pp. 307-318, 1985.

[22] D. M. Sloan, "Fourier pseudospectral solution of the regularised long wave equation," Journal of Computational and Applied Mathematics, vol. 36, no. 2, pp. 159-179, 1991.

[23] A. H. A. Ali, "Chebyshev collocation spectral method for solving the RLW equation," International Journal of Nonlinear Science, vol. 7, no. 2, pp. 131-142, 2009.

[24] K. R. Raslan, "A computational method for the regularized long wave (RLW) equation," Applied Mathematics and Computation, vol. 167, no. 2, pp. 1101-1118, 2005.

[25] D. Bhardwaj and R. Shankar, "Computational method for regularized long wave equation," Computers and Mathematics with Applications, vol. 40, no. 12, pp. 1397-1404, 2000.

[26] S. I. Zaki, "Solitary waves of the splitted RLW equation," Computer Physics Communications, vol. 138, no. 1, pp. 80-91, 2001.

[27] B. Saka and I. Dağ, "Quartic B-spline collocation algorithms for numerical solution of the RLW equation," Numerical Methods for Partial Differential Equations, vol. 23, no. 3, pp. 731-751, 2007.

[28] I. Dağ, B. Saka, and D. Irk, "Galerkin method for the numerical solution of the RLW equation using quintic B-splines," Journal of Computational and Applied Mathematics, vol. 190, no. 1-2, pp. 532-547, 2006.

[29] B. Saka, I. Dağ, and D. Irk, "Quintic B-spline collocation method for numerical solution of the RLW equation," ANZIAM Journal, vol. 49, no. 3, pp. 389-410, 2008.

[30] D. Irk, "Solitary wave solutions for the RLW equation," Physics of Wave Phenomena, vol. 20, pp. 174-183, 2012.

[31] A. Araújo and A. Durán, "Error propagation in the numerical integration of solitary waves. The regularized long wave equation," Applied Numerical Mathematics, vol. 36, no. 2-3, pp. 197217, 2001.

[32] J. Cai, "A new multisymplectic scheme for the RLWequation," Journal of Mathematical Physics, vol. 50, no. 1, p. 16, 2009.

[33] J. Stoer and R. Bulirsch, Introduction To Numerical Analysis, Springer, New York, NY, USA, 1979.

[34] R. E. Mickens, "Nonstandard finite difference schemes for differential equations," Journal of Difference Equations and Applications, vol. 8, no. 9, pp. 823-847, 2002.

[35] R. E. Mickens, "Discretizations of nonlinear differential equations using explicit nonstandard methods," Journal of Computational and Applied Mathematics, vol. 110, no. 1, pp. 181-185, 1999.

[36] R. Anguelov and J. M.-S. Lubuma, "Nonstandard finite difference method by nonlocal approximation," Mathematics and Computers in Simulation, vol. 61, no. 3-6, pp. 465-475, 2003.

[37] R. Anguelov and J. M.-S. Lubuma, "Contributions to the mathematics of the nonstandard finite difference method and applications," Numerical Methods for Partial Differential Equations, vol. 17, no. 5, pp. 518-543, 2001.

[38] R. E. Mickens, "Exact solutions to a finite-difference model of a nonlinear reaction-advection equation: implications for numerical analysis," Journal of Difference Equations and Applications, vol. 9, no. 11, pp. 995-1006, 2003.
[39] R. E. Mickens, "Nonstandard finite difference schemes for reaction-diffusion equations," Numerical Methods for Partial Differential Equations, vol. 15, no. 2, pp. 201-214, 1999.

[40] P. M. Jordan, "A nonstandard finite difference scheme for nonlinear heat transfer in a thin finite rod," Journal of Difference Equations and Applications, vol. 9, no. 11, pp. 1015-1021, 2003.

[41] R. E. Mickens, "A nonstandard finite difference scheme for a fisher PDE having nonlinear diffusion," Computers and Mathematics with Applications, vol. 45, no. 1-3, pp. 429-436, 2003.

[42] R. E. Mickens and P. M. Jordan, "A positivity-preserving nonstandard finite difference scheme for the damped wave equation," Numerical Methods for Partial Differential Equations, vol. 20, no. 5, pp. 639-649, 2004.

[43] R. F. Warming and B. J. Hyett, "The modified equation approach to the stability and accuracy analysis of finite-difference methods," Journal of Computational Physics, vol. 14, no. 2, pp. 159-179, 1974.

[44] F. R. Villatoro and J. I. Ramos, "On the method of modified equations. I: asymptotic analysis of the Euler forward difference method," Applied Mathematics and Computation, vol. 103, no. 2-3, pp. 111-139, 1999.

[45] F. R. Villatoro and J. I. Ramos, "On the method of modified equations. II: numerical techniques based on the equivalent equation for the Euler forward difference method," Applied Mathematics and Computation, vol. 103, no. 2-3, pp. 141-177, 1999.

[46] F. R. Villatoro and J. I. Ramos, "On the method of modified equations. III. Numerical techniques based on the second equivalent equation for the Euler forward difference method," Applied Mathematics and Computation, vol. 103, no. 2-3, pp. 179-212, 1999.

[47] F. R. Villatoro and J. I. Ramos, "On the method of modified equations. IV. Numerical techniques based on the modified equation for the Euler forward difference method," Applied Mathematics and Computation, vol. 103, no. 2-3, pp. 213-240, 1999.

[48] M. Junk and Z. Yang, "Asymptotic analysis of finite difference methods," Applied Mathematics and Computation, vol. 158, no. 1, pp. 267-301, 2004.

[49] F. Rus and F. R. Villatoro, "Numerical methods based on modified equations for nonlinear evolution equations with compactons," Applied Mathematics and Computation, vol. 204, no. 1, pp. 416-422, 2008. 


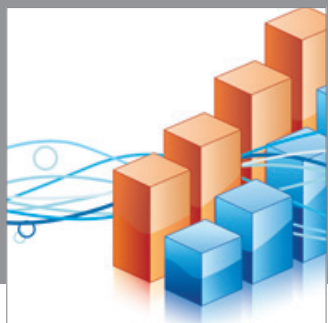

Advances in

Operations Research

mansans

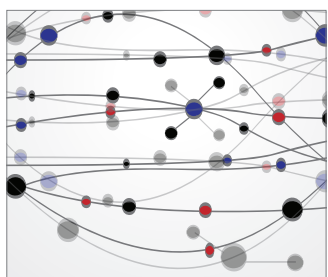

The Scientific World Journal
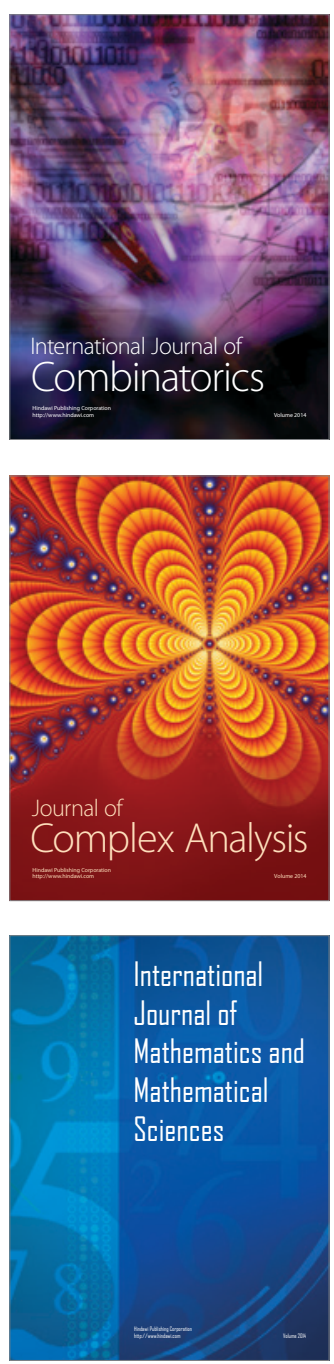
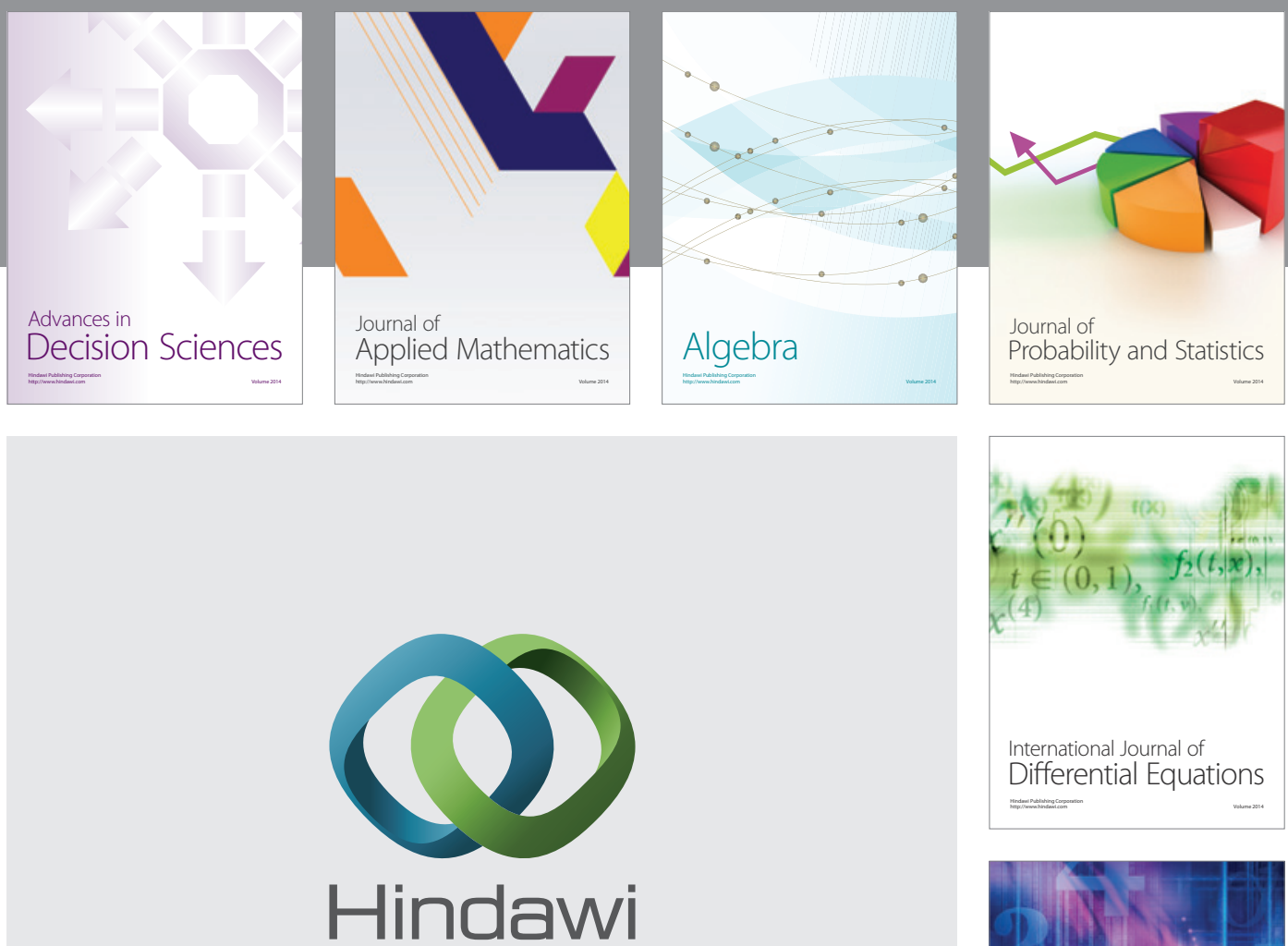

Submit your manuscripts at http://www.hindawi.com
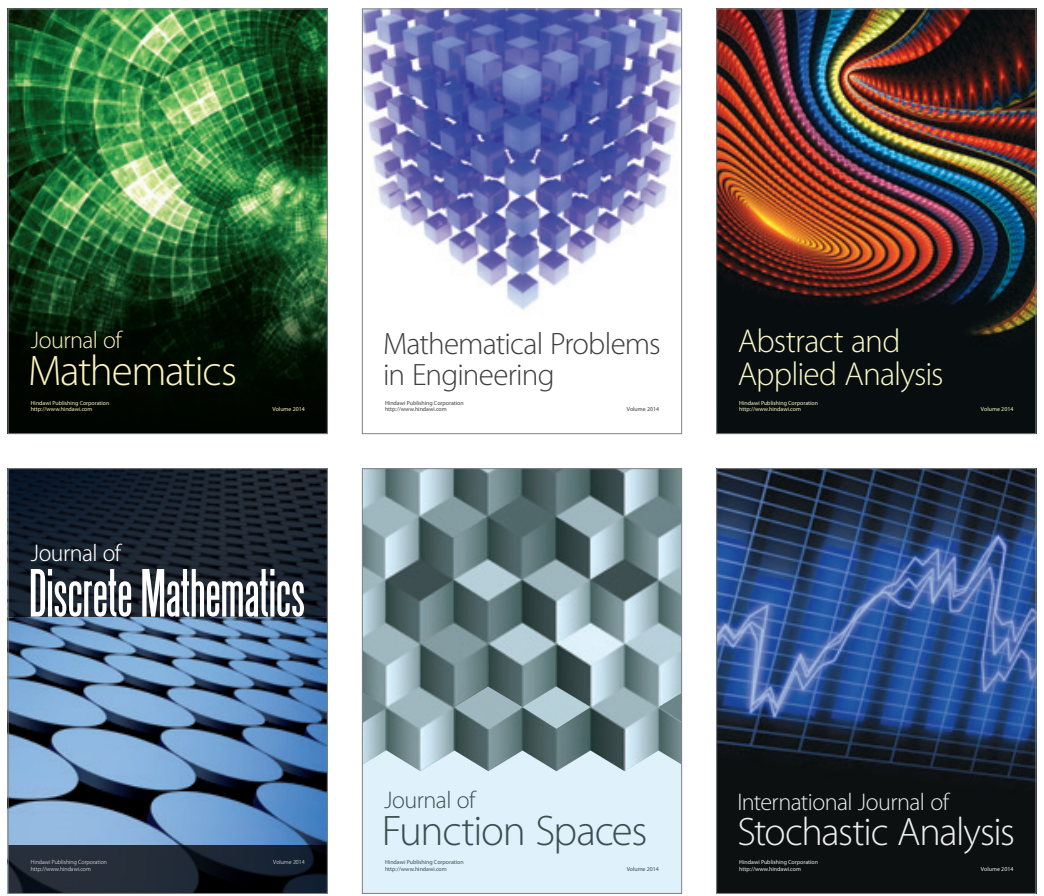

Journal of

Function Spaces

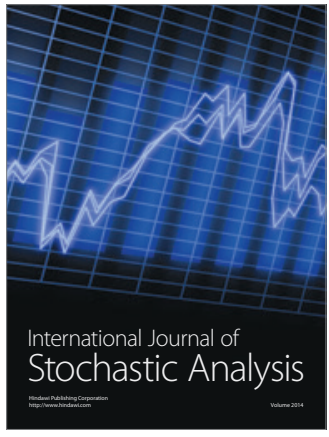

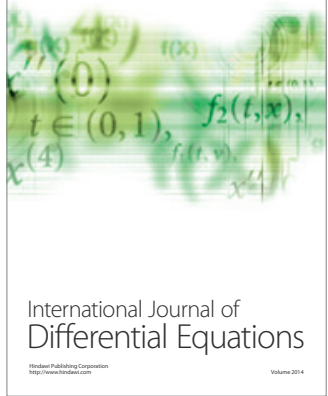
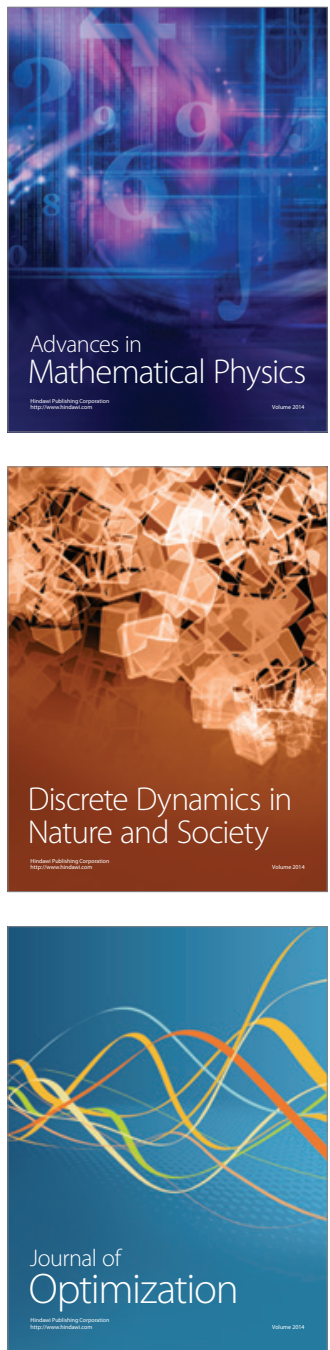